\title{
LightWEIGHT EdUCATIONAL SCARA Robot FOR PAlletizing of USB Sticks
}

\author{
Kemajl Stuja, Mirlind Bruqi, Erich Markl \& Mohamed Aburaia
}
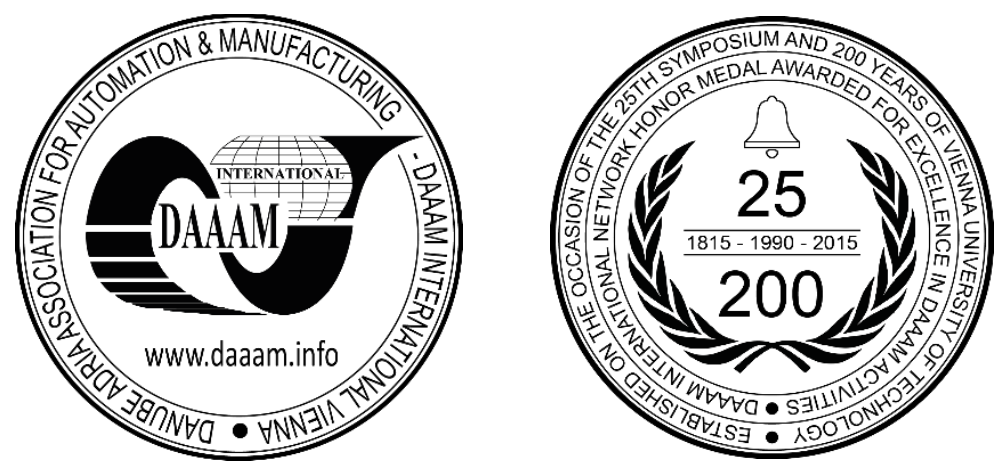

This Publication has to be referred as: Stuja, K[emajl]; Bruqi, M[irlind]; Markl, E[rich] \& Aburaia, M[ohamed] (2016). Lightweight Educational Scara Robot for Palletizing of USB Sticks, Proceedings of the 27th DAAAM International Symposium, pp.0102-0108, B. Katalinic (Ed.), Published by DAAAM International, ISBN 978-3-902734-08-2, ISSN 1726-9679, Vienna, Austria

DOI: $10.2507 / 27$ th.daaam.proceedings.015

\begin{abstract}
This paper deals with design of customized lightweight SCARA- manipulator for educational purposes. In addition to this task shows this paper one concept solution for palletizing of small pieces like USB- Sticks using lightweight Scara. Design and optimization of the mechanical structure was accomplished using the powerful CAE tool -SolidWorks from Dassault Systèmes. Robot parts are generated using additive manufacturing technology- laser sintering of polyamide. The core part of robot controller consist of Arduino- card. Programming will be done using simple editor, which translates a moving and logical instructions of robot into Arduino real time instructions. The vision system mounted at the stand will be responsible for recognition of work-pieces, sending a key information's about the position and orientation of unsorted parts on the incoming conveyor. The Robot pick up the parts and places in ongoing trays into second conveyor. The results of this problem are shown and concisely discussed in this paper. Further work will be focused on developing HMIhuman machine interface for using small devices, such are mobile phones and tablets, to jog and programme the robot targets.
\end{abstract}

Keywords: Scara Robot; CAD; Simulation; Motion Control and Palletizing.

\section{Introduction}

The modern production systems are inclined for custom-made solutions. In the past customized solutions are accompanied with very high engineering costs and very long setup time. Nowadays with the support of a powerful computer design software and advanced manufacturing systems it is possible to manufacture and implement components into production systems. Additive manufactured manipulator controlled with low cost controller card besides the educational purpose will be enough accurate to palletize small parts like a USB-Stick. The main task of the manipulator is to pick up the unsorted USB- Sticks from an input conveyor and to place this into trays - output conveyor. The distance between the two conveyors is about $350 \mathrm{~mm}$ at the same level.

This work shows stage by stage a design of the robot system as well as its integration to the palletizing cell. As mentioned before for this stage of the research a CAE tool SolidWorks was used. The results of this work confirmed that the designed and manufactured robot system under certain circumstances can be used not only in the education but as far for accomplishing a simple manufacturing tasks. The main advantage of this robot is the light weight and the small moment of inertia. So the fast acceleration and short retardation of the robot joints are very significant compared to the 
heavy casting robot. The energy consumption is the fundamental feature of this robot system. The main disadvantage of this robot is the rigidity compared to heavy casting robot.

In the previous work [1] a small 4 axis serial kinematic polyamide robot for palletizing handy covers, was presented. The main disadvantage of this robot- caused by serial kinematic- was vibrations and an elongated time for stabilisation of manipulator, when a high speed was required. In the second work [2] was designed a serial Research Robot for Didactical Development with 6 DoF. The main advantages of this robot can be described as:

- Low- Cost

- Lightweight

- Open hardware and software architecture

- Modular Structure

- Safe use

- Easy to disassemble, in order to explain the internal Structure of the Robot to students etc.

Open hardware and software architecture enables the possibility for mechatronic students to research and develop the future modular structures and the motion control algorithms. In the figure 1 a) was shown the CAD Model of palletizing robot. Figure $1 \mathrm{~b}$ ) shows the 6 axis didactical vertically knick arm robot.
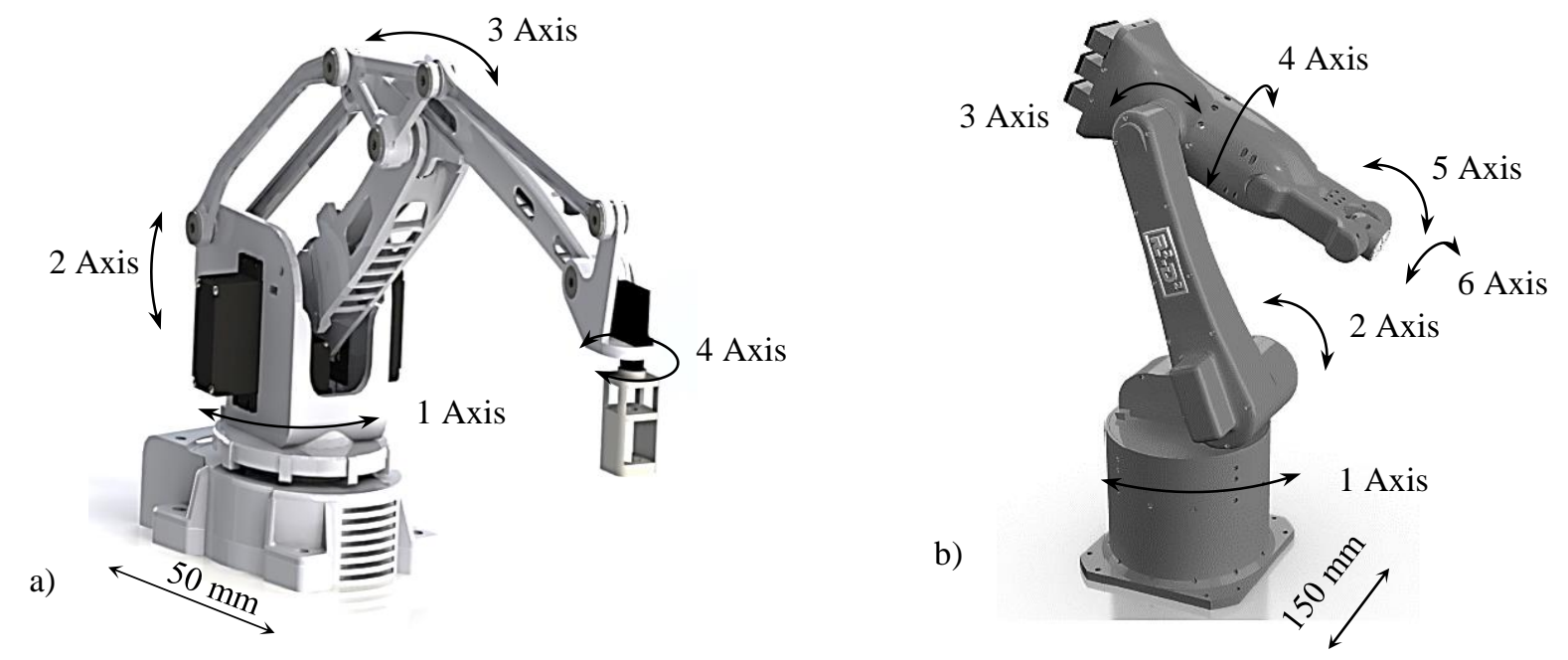

Fig. 1. Education Robots a) 4 DoF Palletizing Robot and b) 6DoF Knickarm Robot

\section{Design of Scara Robot}

Robot links are made by using of the plastic laser sintering system EOS FORMIGA P 110. This Machine uses laser to sinter powdered material, binding it together to create a solid structure of the parts. The slice unit of this platform is about $0.1 \mathrm{~mm}$ (layer thicknesses). The used powder material is PA 2200 which is a non-filled powder on basis of PA 12 with the following characteristics [3]:

- High strength /stiffness.

- good chemical resistance,

- excellent long-term constant behavior,

- high selectivity and detail resolution,

- Various finishing possibilities etc.

\subsection{Mechanical Design of the prior 3D Draft}

The first step, which is necessary to be done, is a drafting of links and Joints of the Scara. Traditionally the procedure starts with the calculation and selection of the motors and gears. Whereas industrial design starts with 3D modelling in order to calculate the motors and the gear. This paper shows the second procedure. Since the robot will be manufactured using additive technology, it is very imported to take care of the feasibility of the 3D printing. The Links which are very long and outside of work area of the 3D Printer, will be fragmented into more parts. This approach is known in the literature as "Designing for manufacturing". The software tool used for 3D modelling was SolidWorks from Dassault Systèmes. SolidWorks covers a wide range in the engineering area such as Mechanical design and optimization, static simulation and dynamic motion analysis. The biggest motor Nema23 of the first joint and second motor Nema 17 of the second joint are placed collinear and bolded to the static part of the robot- to base. The last two motors Nema 11 concerns 
rotation and translation of the last link of the robot. The picture 2 b) shows the kinematic structure of the 4 axis Scara RRR T (R- Rotational joint, T- Translational joint) and the main dimensions.

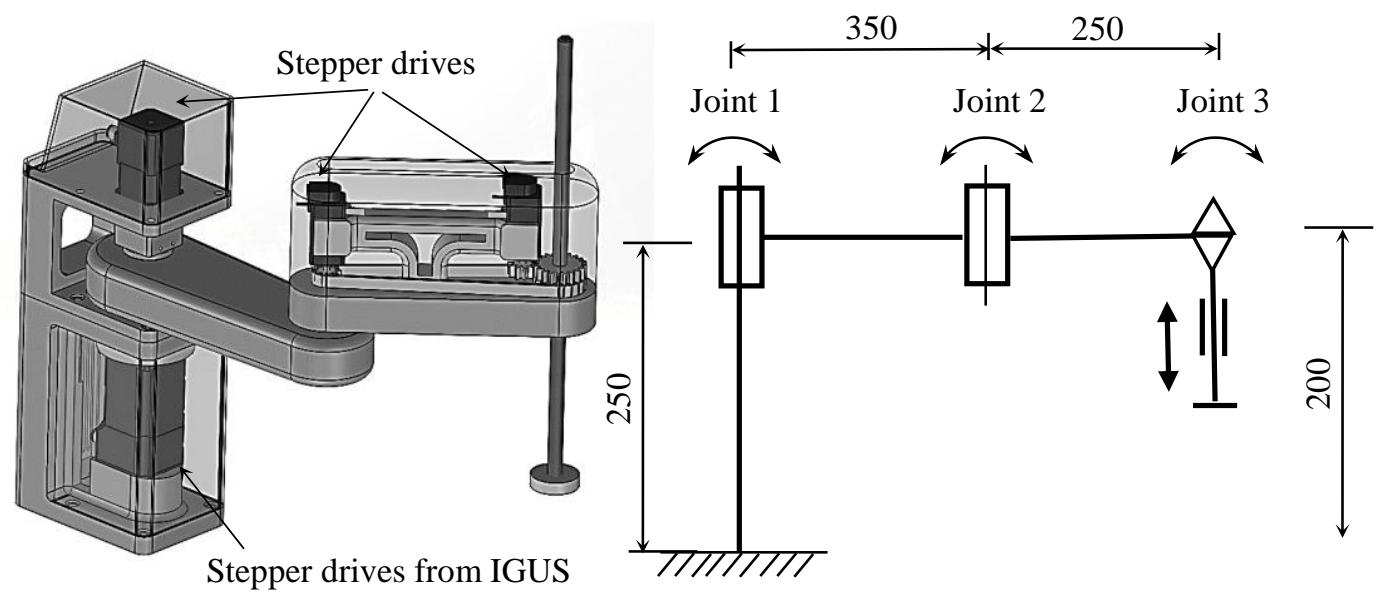

Fig. 2. Scara robot a) CAD design and position of drives, b) kinematic structure of the Scara

\subsection{Drive calculation for the cycle time}

Traditional drive calculations procedure starts with moving profile for the cycle time. In order to achieve the cycle time all drives must ensure the required moment of torque. As shown in the figure below every joint of the robot has three moving stages: acceleration, constant speed and retardation. Following this when the angular velocity is constant the Torque is Zero. In fact for the acceleration and retardation it's very important to calculate the moment of inertia [3].
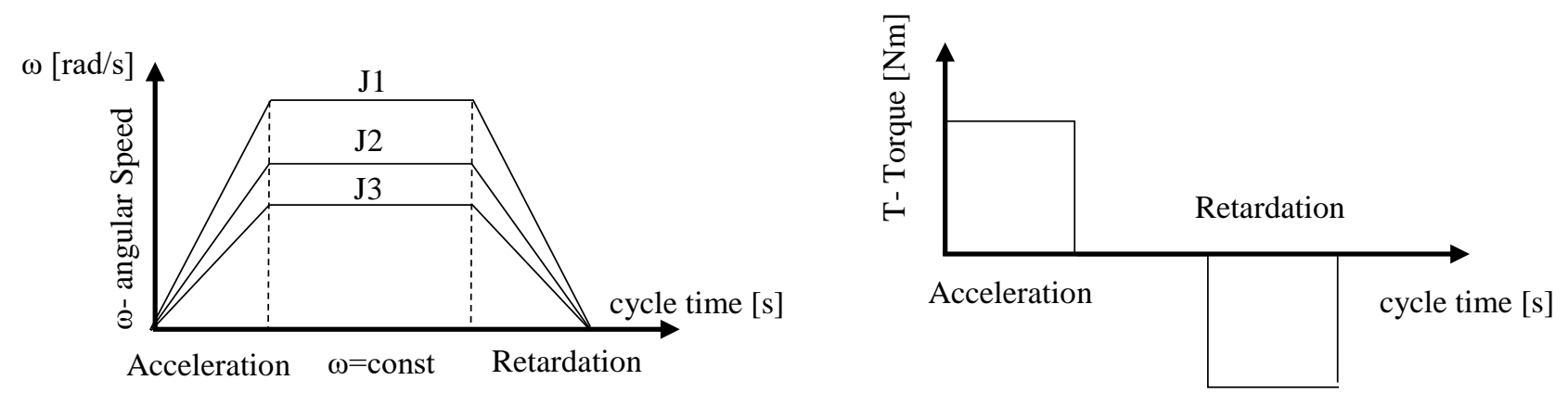

Fig. 3. Movement profile a) The first three joints of SCARA, b) torque

In order to select appropriate motor-gear system or drive system a torque must to be considered:

$$
M=I * \varepsilon
$$

Where: $M$-Torque [Nm], $I$ - inertia [ $\left.\mathrm{kgm}^{2}\right]$ and $\varepsilon$ angular acceleration $\left[\mathrm{rad} / \mathrm{sec}^{2}\right]$.

The second argument is angular acceleration. Cycle time required in this work for pick and place operation was two seconds. That means angular acceleration can be calculated easily from moving profile. A fist argument is mass moment of inertia. Calculation takes very long time, if no CAD system is being used. The more complex the geometry is, the more complex is the calculation. Using CAD system is only a matter of a few clicks. Mass moment of inertia is defined like:

$$
I=\sum_{i=1}^{n} r_{i}^{2} d m_{i}
$$

$r[\mathrm{~m}]$ is the distance from the centre of gravity and rotation axis is usually expressed in meter and $m$ is the mass expressed in kilogram. It is apparent from the (2) formula, that not only the mass of robot parts is a critical parameter for the calculation of the system. A very importing parameter is the distance from the rotation axis. For this reason the second motor was placed to the fixed base in order to reduce the mass of the system and to displace the centre of gravity near to the first link. Secondly, using the PA 2200 material allows to reduce the mass of robot and to take care of energy 
consumptions. The figure 4 a) is a given a snapshot using 3D CAD system SolidWorks for calculation of the centre of gravity and moment of inertia tensor. In figure $4 \mathrm{~b}$ ) is the simulation of the first joint in SolidWorks is shown. For the acceleration of the rest of the system together with end-effector and work piece was needed approximately about 2 $[\mathrm{Nm}]$.Meaning that the drive system must be selected for this quantity in order to fulfil cycle time requirements from the research demands, for the worst case scenario situation.
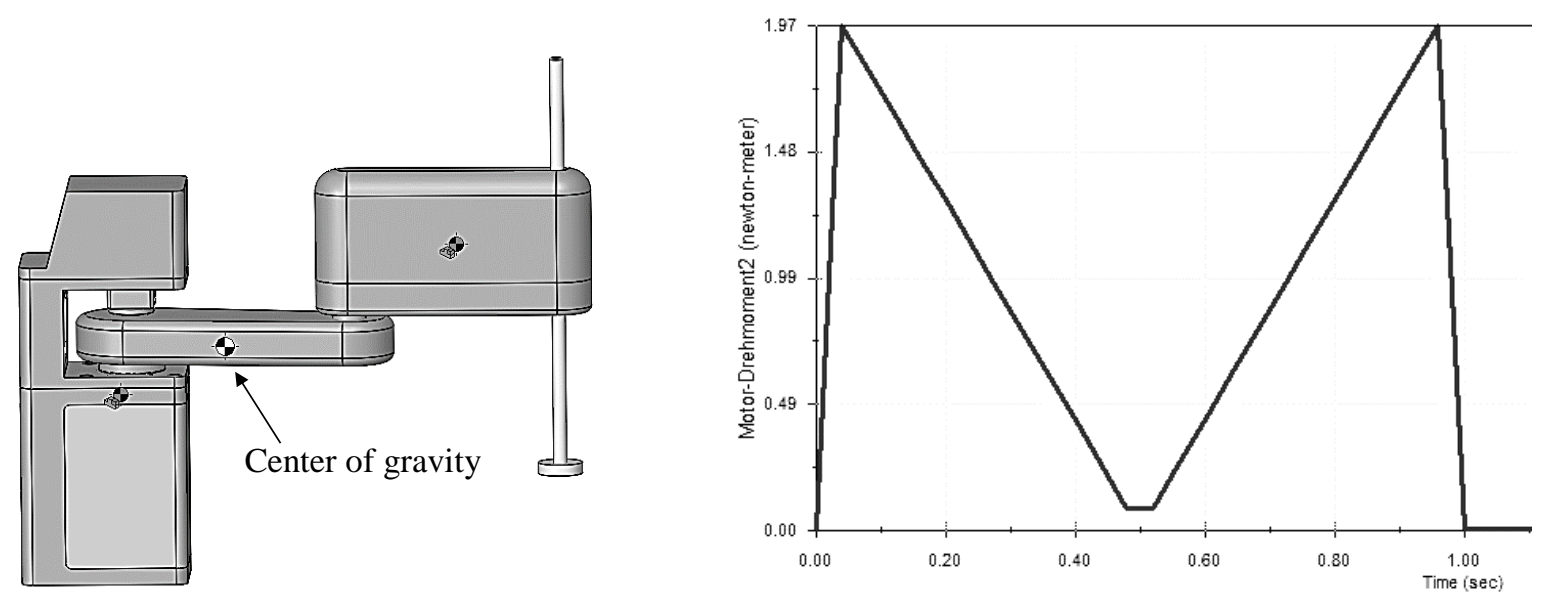

Fig. 4. Scara robot a) CAD design, b) kinematic structure of the Scara

\subsection{Robot kinematics}

Primarily the inverse kinematics was solved. Inverse kinematics deals with the problem of finding the required joint angles to produce a certain desired position and orientation of the end-effector. Finding the inverse kinematics solution for a general manipulator can be a very tricky task. Generally they are non-linear equations. Close-form solutions may not be possible and multiple, infinity, or impossible solutions can arise. Nevertheless, special cases have a closed-form solution and can be solved [3].

For solving the robot kinematics, a very simple approach has been followed. By simplifying the 3 dimension problematic into plan problem ( $\mathrm{x}, \mathrm{y}$ coordinates) and using Pythagoras and Law of cosines to find the position for the first and the second axis. After that the shift in the third demining (z-coordinate)
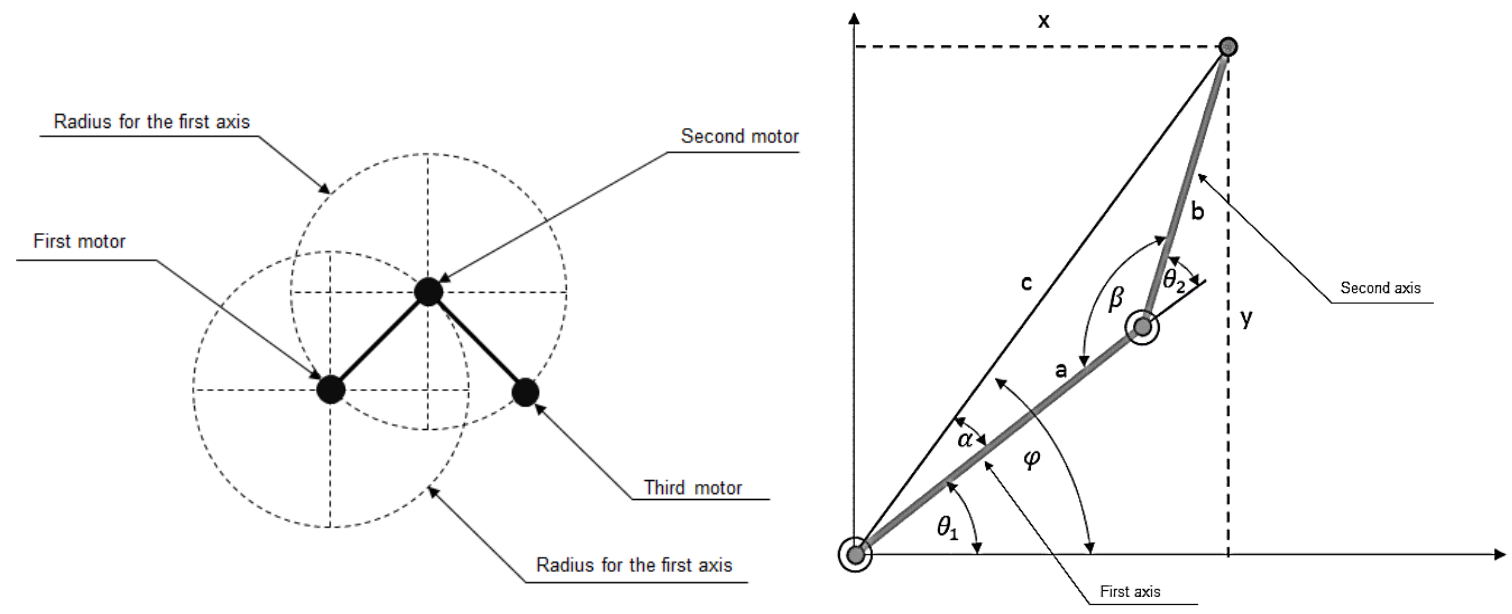

Fig. 6. Solving the inverse kinematics for the first and second axis using Pythagoras and Law of cosines

Finding $\theta_{2}$ - angle for the second joint of robot:

$$
\begin{aligned}
& c^{2}=x^{2}+y^{2} \\
& c^{2}=a^{2}+b^{2}-2 a b \cos (\beta) \\
& \beta=180-\theta_{2} \rightarrow \cos (\beta)=\cos \left(180-\theta_{2}\right)=-\cos \left(\theta_{2}\right)
\end{aligned}
$$


$\theta_{2}=\arccos \left(\frac{-a^{2}-b^{2}+c^{2}}{2 a c}\right)$

why $c=\sqrt{x^{2}+y^{2}}$, then

$\theta_{2}=\arccos \left(\frac{-a^{2}-b^{2}+x^{2}+y^{2}}{2 a \sqrt{x^{2}+y^{2}}}\right)$

Finding $\theta_{1}$ - angle for the first joint of robot:

$$
\begin{aligned}
& b^{2}=a^{2}+c^{2}-2 \operatorname{accos}(\alpha) \\
& \alpha=\operatorname{arcos}\left(\frac{a^{2}-b^{2}+c^{2}}{2 a c}\right) \\
& \alpha=\operatorname{arcos}\left(\frac{a^{2}-b^{2}+x^{2}+y^{2}}{2 a \sqrt{x^{2}+y^{2}}}\right) \\
& \theta_{1}=\varphi-\alpha \\
& \varphi=\arctan \left(\frac{y}{x}\right) \\
& \theta_{1}=\left(\arctan \left(\frac{y}{x}\right)-\arccos \left(\frac{a^{2}-b^{2}+x^{2}+y^{2}}{2 a \sqrt{x^{2}+y^{2}}}\right)\right)
\end{aligned}
$$

\subsection{Robot Control System}

Stepper motors have found their way into many different areas of automation and control systems. The wide popularity of these motors can be attributed in part to the various ways the motor can be driven and because of its compatibility with digital systems. In particular, stepper motors are ideal for control systems that require discrete, easily repeatable movements at moderate to low frequencies. The figure below shows an example block diagram of a system with microcontroller, stepper motor, and encoder- feedback. In this research, stepper motors with a feedback provided for precision assistance will be used [4].

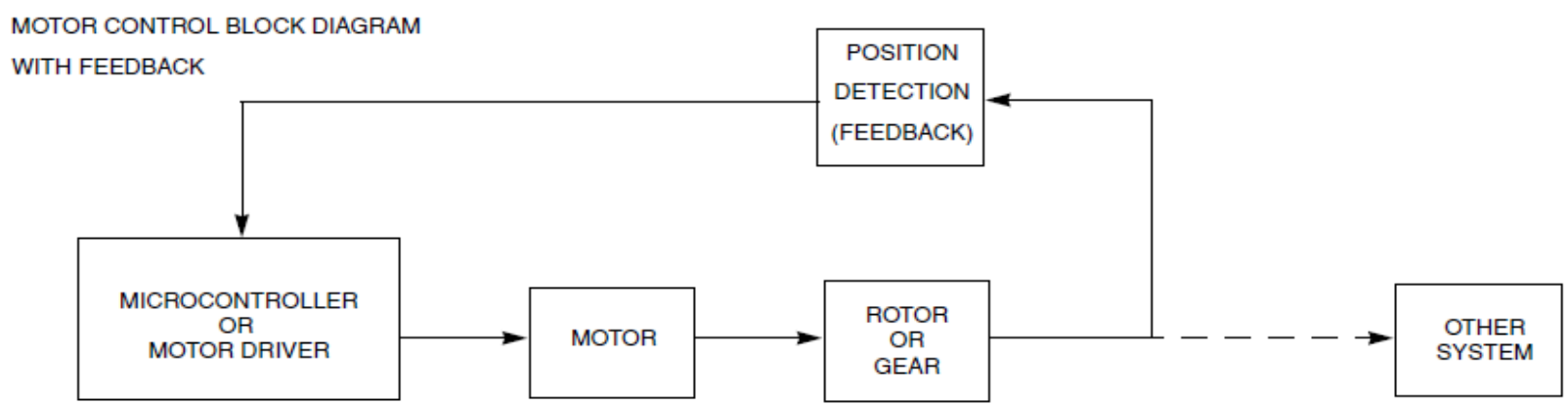

Fig. 6. Example System with a Microcontroller, Stepper Motor, and Feedback [4]

For controlling the robot system, the open Open-source electronic prototyping platform Arduino will be used. Simulink (the add-on product to MATLAB) provides an interactive, graphical environment for modelling, simulating, and analysing of dynamic systems. It enables rapid construction of virtual prototypes to explore design concepts at any level of detail with minimal effort [5]. Simulink has also a support package for Arduino hardware, which allows users to develop and simulate algorithms that run standalone on the Arduino and configuring and accessing the sensors and actuators on Arduino [7]. The following figure shows how to use the Stepper Motor Driver and Stepper Motor blocks together to implement a controlled permanent magnet stepper motor with Simulink [6]. 


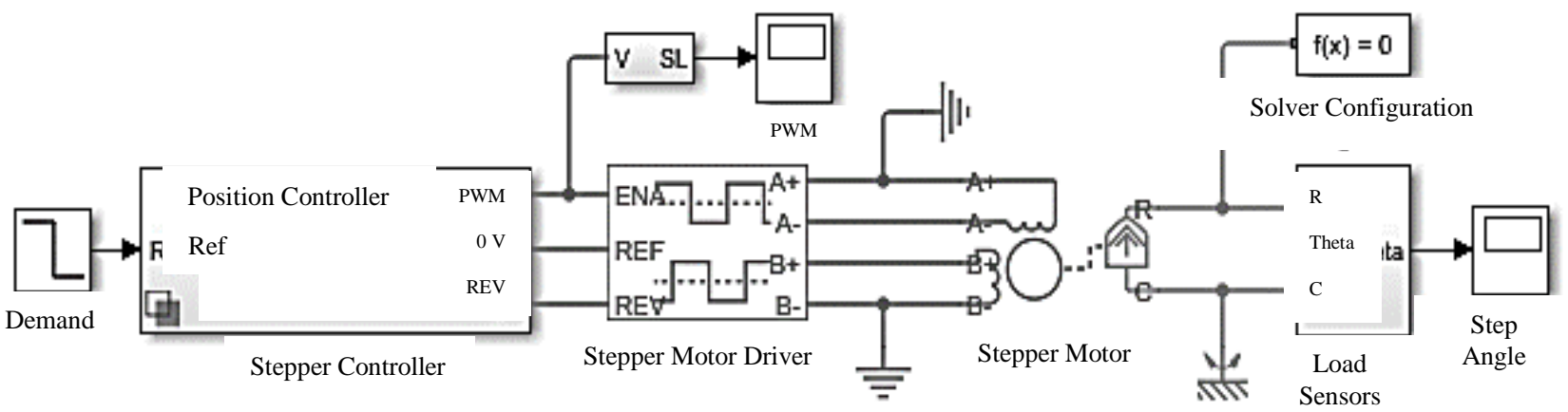

Fig. 7. Controlling a stepper motor with Simulink [6]

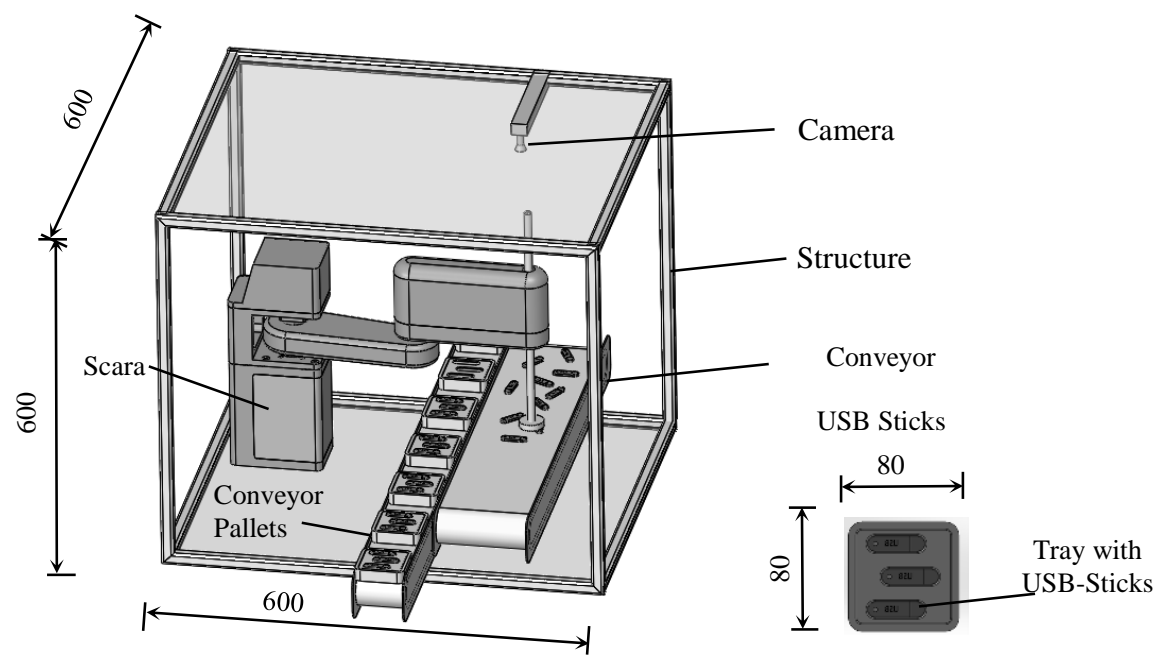

Fig. 8. Layout of robot cell for palletizing of USB-Sticks

\section{Conclusion}

The goal of this research was to design and manufacturing robot system for educational purposes and to improve a performance of this system for by simple manufacturing task, such is palletizing of USB- Sticks (see the Figure 8). The design stage was done using the powerful CAD tool- SolidWorks. This powerful tool reduced radically the engineering effort and provided a possibility for the optimization of robot mechanical structure. In this perspective was reduced the weight of manipulator converting them into lightweight robot class. Lightweight ability of robot allows the fast acceleration as well as short the breaking time. The energy consumption per cycle time was far below comparing him with industrial robot. The outcomes of this research confirmed our statement, which for the simple manufacturing tasks and moderate accuracy, additive manufactured robots provides passable solution.

However this work left some opened issues and future works to be done. The future works are focused in optimizing the structure of robots and human machine interface. The heavy step motors will be replaced by lightweight servo motors in order to reduce the mass of the robot as well for better positioning / path accuracy. By using of high resolution of encoders it will be possible to capture the vibrations in order to counteract with control logic. The second task will be focused on developing HMI- human machine interface. It will be possible to use the tablets and small devices like mobile phones to jog and programme the robot targets.

\section{Acknowledgments}

We would like to express our special thanks of gratitude to IGUS- Company- Austria for sponsoring the key components of the Scara robot. Without this support it would not be possible to realise this research inside the planed period. IGUS produces long life industrial components such as cables, connectors, bearings and joints-links for small robots. 


\section{References}

[1] Aburaia, B.; Markl, E. \& Stuja, K. (2014). New concept for design and control of 4 axis robot using the additive manufacturing technology, 25th DAAAM International Symposium on Intelligent Manufacturing and Automation, Elsevier, ISSN 1877-7058, Katalinic, B. (Ed.), pp. 1364-1369, Vienna.

[2] Schweidler, R.; Aburaia, M. \& Engelhardt-Nowitzki, C. (2016). Design of an Industrial Robot with Six Degrees of Freedom for Educational Purposes, OAGM \& ARW joint workshop on "Computer Vision and Robotics" Kurt Niel, Peter M. Roth, Markus Vincze. (Ed.), pp. 155-161, Wels, Austria

[3] Pires, J. N. (2007), Robot Manipulators and Control Systems, ISBN: 978-0-387-23325-3, Publisher: Springer US, New York, DOI 10.1007/b101252.

[4] http://pro.sculpteo.com (2014), PA2200 Datasheet, Fine Polyamide PA 2200 for EOSINT P, Available at: http://pro.sculpteo.com/media/data/faq/pa2200, Accessed: 2014-09-20.

[5] Matthew Grant, (2005). Quick Start for Beginners to Drive a Stepper Motor, Available from: http://www.nxp.com/files/microcontrollers/doc/app_note/AN2974.pdf Accessed: 2016-09-20.

[6] https://ewh.ieee.org (2016), IEEE- Institute of Electrical and Electronics Engineers, New York, Available from: https://ewh.ieee.org/r1/ct/sps/PDF/MATLAB/chapter8.pdf, Accessed: 2016-09-20.

[7] http://de.mathworks.com (2016), MathWorks, Natick, Massachusetts, USA Available from: http://de.mathworks.com/ help/physmod/elec/ examples/controlled-stepper-motor.html, Accessed: 2016-09-20.

[8] http://de.mathworks.com (2016), MathWorks, Natick, Massachusetts, USA Available from: http://de.mathworks .com/hardware-support/arduino-simulink.html?requestedDomain=de.mathworks.com, Accessed: 2016-09-20. 